\title{
Existence and uniqueness theorems for fourth-order boundary value problems
}

\author{
by Jolanta PRzybycin (Kraków)
}

\begin{abstract}
We establish the existence and uniqueness theorems for a linear and a nonlinear fourth-order boundary value problem. The results obtained generalize the results of Usmani [4] and Yang [5]. The methods used are based, in principle, on [3], [5].
\end{abstract}

1. Let $\mathcal{L}$ be a differential operator of the form $\mathcal{L}=\mathcal{L}_{1} \circ \mathcal{L}_{0}$, where $\mathcal{L}_{i}$ denotes the Sturm-Liouville operator defined by $\mathcal{L}_{i} y=-\left(p_{i} y^{\prime}\right)^{\prime}+q_{i} y$, $i=0,1$. As usual we assume $p_{i} \in C^{3-2 i}[0,1], q_{i} \in C^{2-2 i}[0,1]$ and $p_{i}>0$, $q_{i} \geq 0$ on $[0,1]$.

Consider the nonlinear problem

$$
\begin{aligned}
& \mathcal{L} y=F(\cdot, y) \quad \text { in }(0,1), \\
& y(0)=y_{0}, \quad y(1)=y_{1}, \quad \mathcal{L}_{0} y(0)=\widehat{y}_{0}, \quad \mathcal{L}_{0} y(1)=\widehat{y}_{1} .
\end{aligned}
$$

Denote the above boundary conditions by (B.C.). By a solution of (1) we understand $u \in C^{4}[0,1] \cap$ (B.C.) satisfying (1).

Usmani studied a particular case of $(1)$, namely $\mathcal{L} y=y^{(4)}$ and $F(x, y)=$ $f(x) y+g(x)$. He proved an existence and uniqueness theorem under the condition $\sup _{x \in[0,1]}|f(x)|<\pi^{4}$. Yang found a better condition on $f$ which guarantees the unique solvability of the above problem, namely $f(x) \neq j^{4} \pi^{4}$ for $j=1,2, \ldots$ He also showed an existence theorem for the nonlinear problem $y^{(4)}=F\left(\cdot, y, y^{\prime \prime}\right)$, (B.C.), under the assumption $|F(x, \xi, \eta)| \leq$ $a|\xi|+b|\eta|+c, a / \pi^{4}+b / \pi^{2}<1$, which is essential to the proof. By applying the result of Yang to $F\left(\cdot, y, y^{\prime \prime}\right)=f(\cdot, y)+q y^{\prime \prime}$, where $q$ is a positive and continuous function on $[0,1]$ we obtain the existence of solution if $a / \pi^{4}+\max _{x \in[0,1]} q(x) / \pi^{2}<1$. This sufficient condition seems to be very restrictive. To illustrate this fact consider the equation $\mathcal{L} y=y^{(4)}-k^{2} \pi^{2} y^{\prime \prime}=0$ with (B.C.). It is easily verified that this problem is uniquely solvable for any $k \in \mathbb{R}$.

1991 Mathematics Subject Classification: Primary 34B15.

Key words and phrases: eigenvalue, Leray-Schauder degree, Fredholm alternative. 
We shall now see that it is possible to find a better condition for $F$ by proving a theorem which is more general than the result of Yang in some respects but less general in other ones.

Theorem 1. Let $p_{i 0}=\min _{x \in[0,1]} p_{i}(x)$ and $q_{i 0}=\min _{x \in[0,1]} q_{i}(x)$. Suppose that $F$ is continuous on $[0,1]$ and satisfies the condition

$$
\exists_{a, b \geq 0, a<\left(\pi^{2} p_{00}+q_{00}\right)\left(\pi^{2} p_{10}+q_{10}\right)} \forall_{(x, \xi) \in[0,1] \times \mathbb{R}} \quad|F(x, \xi)| \leq a|\xi|+b .
$$

Then for every $y_{0}, y_{1}, \widehat{y}_{0}, \widehat{y}_{1} \in \mathbb{R}$ problem (1) has a solution.

This result may be proved in much the same way as the theorem of Yang. The main tool of the proof is the classical method of a priori bounds. Let us introduce the family of problems

$$
\begin{aligned}
& \mathcal{L} y=t F(\cdot, y) \quad \text { in }(0,1), \\
& y(0)=t y_{0}, \quad y(1)=t y_{1}, \quad \mathcal{L}_{0} y(0)=t \widehat{y}_{0}, \quad \mathcal{L}_{0} y(1)=t \widehat{y}_{1} .
\end{aligned}
$$

Denote by (, ) the scalar product and by \|\| the norm in $L^{2}(0,1)$. The next theorem will provide a priori estimates for solutions of $\left(1_{t}\right)$.

Theorem 2. Let $y_{t}$ denote a solution of $\left(1_{t}\right)$. Then

$$
\exists M>0 \forall t \in[0,1] \quad\left\|y_{t}\right\|+\left\|\mathcal{L}_{0} y_{t}\right\| \leq M .
$$

Pr o of. Choose a smooth function $w:[0,1] \rightarrow \mathbb{R}$ satisfying the boundary conditions $w(0)=y_{0}, w(1)=y_{1}, \mathcal{L}_{0} w(0)=\widehat{y}_{0}, \mathcal{L}_{0} w(1)=\widehat{y}_{1}$. Let $z_{t}=$ $y_{t}-t w$. Setting $G(x, z(x))=t F(x, z(x)+t w(x))-t \mathcal{L} w(x)$, we see that $z_{t}$ satisfies the equation

$$
\begin{aligned}
& \mathcal{L} z=G(\cdot, z) \quad \text { in }(0,1), \\
& z(0)=z(1)=\mathcal{L}_{0} z(0)=\mathcal{L}_{0} z(1)=0 .
\end{aligned}
$$

From (2) we have $|G(x, \xi)| \leq a|\xi|+b_{1}$, where $b_{1}$ depends on $b$ and $w$. Setting $u=\mathcal{L}_{0} z$ we can study the following coupled problem:

$$
\begin{array}{ll}
\mathcal{L}_{0} z=u, & z(0)=z(1)=0, \\
\mathcal{L}_{1} u=G(\cdot, z), & u(0)=u(1)=0 .
\end{array}
$$

By applying the Schwarz inequality combined with the Poincaré inequality we have the estimate

$$
\begin{aligned}
\left(p_{00} \pi^{2}+q_{00}\right)\|z\|^{2} & \leq p_{00}\left\|z^{\prime}\right\|^{2}+q_{00}\|z\|^{2} \leq \int_{0}^{1}\left(p_{0}(x)\left[z^{\prime}(x)\right]^{2}+q_{0}(x)[z(x)]^{2}\right) d x \\
& =\left(\mathcal{L}_{0} z, z\right)=(u, z) \leq\|u\| \cdot\|z\| .
\end{aligned}
$$

Hence

$$
\|z\| \leq \frac{1}{p_{00} \pi^{2}+q_{00}}\|u\|
$$


Proceeding analogously we obtain for arbitrary $\varepsilon>0$,

$$
\begin{aligned}
\left(p_{10} \pi^{2}+q_{10}\right)\|u\|^{2} & \leq\left(u, \mathcal{L}_{1} u\right)=(u, G(\cdot, z)) \\
& \leq \int_{0}^{1}\left(a|u(x)| \cdot|z(x)|+b_{1}|u(x)|\right) d x \\
& \leq a\|u\| \cdot\|z\|+\frac{1}{2} \varepsilon\|u\|^{2}+\frac{b_{1}^{2}}{2 \varepsilon} \\
& \leq\left(\frac{a}{p_{00} \pi^{2}+q_{00}}+\frac{1}{2} \varepsilon\right)\|u\|^{2}+\frac{b_{1}^{2}}{2 \varepsilon} .
\end{aligned}
$$

Since $a$ satisfies (2) we can choose $\varepsilon$ sufficiently small such that

Hence

$$
1-\frac{a}{\left(p_{00} \pi^{2}+q_{00}\right)\left(p_{10} \pi^{2}+q_{10}\right)}-\frac{\varepsilon}{2\left(\pi^{2} p_{10}+q_{10}\right)}=k>0 .
$$

and consequently

$$
\|u\| \leq \frac{b_{1}}{\left[2 \varepsilon k\left(p_{10} \pi^{2}+q_{10}\right)\right]^{1 / 2}}=b_{2}
$$

Thus the proof is complete.

$$
\|z\| \leq \frac{b_{2}}{p_{00} \pi^{2}+q_{00}} .
$$

Proof of Theorem 1. Problem $\left(1_{t}\right)$ can be written in the form

$$
\begin{array}{ll}
\mathcal{L}_{0} y=u, & y(0)=t y_{0}, y(1)=t y_{1}, \\
\mathcal{L}_{1} u=t F(\cdot, y), & u(0)=t \widehat{y}_{0}, u(1)=t \widehat{y}_{1} .
\end{array}
$$

Let $G_{i}$ for $i=0,1$ be the Green function of the equation $\mathcal{L}_{i} v=h$ in $(0,1)$, with $v(0)=v(1)=0$. Then $v(x)=\int_{0}^{1} G_{i}(x, s) h(s) d s$. Using $G_{i}$ we can transform $\left(1_{t}^{\prime}\right)$ into the equivalent system of integral equations

$$
\begin{aligned}
& y(x)=t y_{0}+x t\left(y_{1}-y_{0}\right)+\int_{0}^{1} G_{0}(x, s) u(s) d s, \\
& u(x)=t \widehat{y}_{0}+x t\left(\widehat{y}_{1}-\widehat{y}_{0}\right)+\int_{0}^{1} t G_{1}(x, s) F(s, y(s)) d s .
\end{aligned}
$$

Let $E=L^{2}(0,1) \times L^{2}(0,1)$. It is a Banach space equipped with the norm $\|(y, u)\|=\|y\|+\|u\|$. Define a map $T_{t}: E \rightarrow E$ by $T_{t}=\left(T_{t}^{0}, T_{t}^{1}\right)$ where $T_{t}^{0}(y, u), T_{t}^{1}(y, u)$ are the right-hand sides of $(*)$ and $(* *)$ respectively. To prove that problem (1) has a $C^{4}$-solution it is enough to search for solutions of $\left(I-T_{1}\right)(y, u)=0$ in $E$. It is easily seen that $T_{t}$ is a compact operator for every $t \in[0,1]$. Thus we see that the Leray-Schauder degree theory applies to $I-T_{t}$ and $t$ is an allowable homotopy parameter. Consider $\bar{B}_{M+1}=\{(y, z) \in E:\|(y, u)\| \leq M+1\}$. The estimate (3) guarantees that 
$\operatorname{deg}\left(I-T_{t}, B_{M+1}, 0\right)$ is well defined for each $t \in[0,1]$ and, by using the homotopy invariance of the degree we have

$$
\operatorname{deg}\left(I-T_{1}, B_{M+1}, 0\right)=\operatorname{deg}\left(I-T_{0}, B_{M+1}, 0\right)=\operatorname{deg}\left(I, B_{M+1}, 0\right)=1 .
$$

Consequently, $\left(I-T_{1}\right)(y, u)=0$ has a solution in $B_{M+1}$, which completes the proof.

Remark 3. Let $\mu_{1}^{i}$ denote the first eigenvalue of the problem $\mathcal{L}_{i} y=\mu y$ subject to $y(0)=y(1)=0$. From the above proof it is clear that using the variational definition of $\mu_{1}^{i}$ we can replace the assumption (2) by

$$
\exists a, b \geq 0, a<\mu_{1}^{0} \mu_{1}^{1} \forall x, \xi \quad|F(x, \xi)| \leq a|\xi|+b .
$$

Remark 4. The equation $\mathcal{L} y=y^{(4)}-3 \pi^{2} y^{\prime \prime}=4 \pi^{4} y$ has no solutions when $y_{0}+y_{1}+\left(1 /\left(4 \pi^{2}\right)\right)\left(\widehat{y}_{0}+\widehat{y}_{1}\right) \neq 0$, which means that assumption $(2)$ is sharp.

2. Let us return to problem (1) in a linear version similar to that which was investigated by Usmani. The function $F$ has the form $F(x, y)=f(x) y+$ $g(x)$, where $f$ and $g$ are continuous on $[0,1]$. So, we consider the problem

$$
\mathcal{L} y=f y+g \quad \text { in }(0,1)
$$

together with the boundary conditions (B.C.). If we assume additionally that the operator $\mathcal{L}$ is symmetric and positive definite (this is satisfied in particular when $\mathcal{L}_{0}=\mathcal{L}_{1}$ ) then the linear problem

$$
\mathcal{L} v=\mu v
$$

together with the boundary conditions

$$
v(0)=v(1)=\mathcal{L}_{0} v(0)=\mathcal{L}_{0} v(1)=0
$$

has an increasing sequence of positive eigenvalues $0<\mu_{1}<\mu_{2}<\ldots$

Our main result for (4) is:

Theorem 5. If $f(x) \neq \mu_{j}, j=1,2, \ldots$, then for any chosen $y_{0}, y_{1}, \widehat{y}_{0}, \widehat{y}_{1}$ and an arbitrary function $g$ problem (4) has a unique solution.

This result may be obtained by applying a mapping theorem for nonlinear operators of the form $L-N$ in a Hilbert space, with $L$ linear and $N$ nonlinear, proved by Mawhin in [3]. Nevertheless, for clarity and simplicity we give the direct proof of Theorem 5 which is based in great part on Mawhin's idea.

Proof of Theorem 5. Using the Green functions introduced in Section 1 we can convert problem (4) into an equivalent integral equation over $C[0,1]$ :

$$
y-T y=h,
$$


where

$$
\begin{aligned}
T y(x)= & \int_{0}^{1} G_{0}(x, s)\left[\int_{0}^{1} G_{1}(s, t) f(t) y(t) d t\right] d s, \\
h(x)= & y_{0}+x\left(y_{1}-y_{0}\right) \\
& +\int_{0}^{1} G_{0}(x, s)\left[\widehat{y}_{0}+s\left(\widehat{y}_{1}-\widehat{y}_{0}\right)+\int_{0}^{1} G_{1}(s, t)(f(t) y(t)+g(t)) d t\right] d s .
\end{aligned}
$$

It is clearly enough to show that (5) is uniquely solvable for arbitrary $h \in$ $C[0,1]$. Since $T$ is a compact operator we can apply the Fredholm alternative. So, it is sufficient to prove that the boundary value problem

$$
\begin{aligned}
& \mathcal{L} y=f y \quad \text { in }(0,1), \\
& y(0)=y(1)=\mathcal{L}_{0} y(0)=\mathcal{L}_{0} y(1)=0,
\end{aligned}
$$

has only the trivial solution. The differential operator $\mathcal{L}$ together with the boundary conditions $y(0)=y(1)=\mathcal{L}_{0} y(0)=\mathcal{L}_{0} y(1)=0$ defines an unbounded selfadjoint operator $L$ in $L^{2}(0,1)$, so that problem (6) can be rewritten as

$$
(L-k I) y=\widehat{F}(y),
$$

where $k \in \mathbb{R}$ and $\widehat{F}$ denotes the operator of multiplication by $f-k$, namely $\widehat{F}(y)(x)=(f(x)-k) y(x)$.

We denote by $\sigma(L)$ the spectrum of $L$. For $k \neq \mu_{j}, L-k I$ is invertible, so that (7) is equivalent to

$$
y=(L-k I)^{-1} \widehat{F}(y) .
$$

Since $\left\|(L-k I)^{-1}\right\|^{-1}=\operatorname{dist}(k, \sigma(L))([2])$, we obtain

$$
\begin{aligned}
\left\|(L-k I)^{-1} \widehat{F}\right\| & \leq\left\|(L-k I)^{-1}\right\| \cdot\|\widehat{F}\| \\
& =\frac{\|\widehat{F}\|}{\operatorname{dist}(k, \sigma(L))} \leq \frac{\max _{x \in[0,1]}|f(x)-k|}{\operatorname{dist}(k, \sigma(L))} .
\end{aligned}
$$

There are two possibilities: either $\max _{x \in[0,1]} f(x)<\mu_{1}$, or there exists $j \in \mathbb{N}$ such that $\mu_{j}<\min _{x \in[0,1]} f(x) \leq \max _{x \in[0,1]} f(x)<\mu_{j+1}$.

Note that

$$
\operatorname{dist}(k, \sigma(L))= \begin{cases}\mu_{1}-k & \text { for } k<\mu_{1}, \\ \inf \left\{k-\mu_{j}, \mu_{j+1}-k\right\} & \text { for } k \in\left(\mu_{j}, \mu_{j+1}\right) .\end{cases}
$$

It is clear that we can choose $k$ depending on $f$ such that $\left\|(L-k I)^{-1} \widehat{F}\right\|<1$. So (7) has only the trivial solution. This completes the proof.

Consider the particular case of problem (4), namely

$$
y^{(4)}=f(x) y+q_{1} y^{\prime \prime}+g(x)
$$


with the boundary conditions (B.C.). The next result is an immediate consequence of Theorem 5 .

Theorem 6. If $f(x) \neq j^{4} \pi^{4}, j=1,2, \ldots$, then for any chosen $y_{0}, y_{1}$, $\widehat{y}_{0}, \widehat{y}_{1}$ and arbitrary functions $g$ and $q_{1}$ problem (8) has a unique solution.

Notice that $y^{(4)}=-\pi^{2} y^{\prime \prime}$ has no solutions when $\widehat{y}_{0}+\widehat{y}_{1} \neq 0$, which shows that the condition $q_{i} \geq 0$ is sharp.

\section{References}

[1] M. S. Berger, Nonlinearity and Functional Analysis, Academic Press, New York, 1977.

[2] T. Kato, Perturbation Theory for Linear Operators, Springer, 1966.

[3] J. Mawhin, Contractive mappings and periodically perturbed conservative systems, Arch. Math. (Brno) 12 (1976), 67-74.

[4] R. A. Usmani, A uniqueness theorem for a boundary value problem, Proc. Amer. Math. Soc. 77 (1979), 329-335.

[5] Y. Yang, Fourth-order two-point boundary value problems, ibid. 104 (1988), 175-180.

Institute of Mathematics

University of Mining and Metallurgy

Mickiewicza 30

30-059 Kraków, Poland 